\title{
Large Solutions of Quasilinear Elliptic System of Competitive Type: Existence and Asymptotic Behavior
}

\author{
Lin Wei $^{1}$ and Zuodong Yang ${ }^{1,2}$ \\ ${ }^{1}$ Institute of Mathematics, School of Mathematics Science, Nanjing Normal University, \\ Jiangsu, Nanjing 210046, China \\ ${ }^{2}$ College of Zhongbei, Nanjing Normal University, Jiangsu, Nanjing 210046, China
}

Correspondence should be addressed to Zuodong Yang, zdyang_jin@263.net

Received 22 July 2009; Accepted 23 October 2009

Academic Editor: Wenming Zou

Copyright (C) 2010 L. Wei and Z. Yang. This is an open access article distributed under the Creative Commons Attribution License, which permits unrestricted use, distribution, and reproduction in any medium, provided the original work is properly cited.

We study the existence and asymptotic behavior of positive solutions for a class of quasilinear elliptic systems in a smooth boundary via the upper and lower solutions and the localization method. The main results of the present paper are new and extend some previous results in the literature.

\section{Introduction}

This paper is concerned with the study of positive boundary blow-up solutions to a quasilinear elliptic system of competitive type:

$$
\begin{gathered}
\Delta_{p} u=a(x) u^{a} v^{b} \quad \text { in } \Omega, \\
\Delta_{p} v=b(x) u^{c} v^{e} \quad \text { in } \Omega, \\
u=v=+\infty \quad \text { on } \partial \Omega,
\end{gathered}
$$

where $\Omega$ is a bounded $C^{2}$ domain of $\mathbf{R}^{\mathbf{N}}$ and $\Delta_{p}$ stands for the $p$-Laplacian operator defined by $\Delta_{p} u=\operatorname{div}\left(|\nabla u|^{p-2} \nabla u\right), p>1$. The exponents $a, b, c, e$ verify $a, e>p-1, b, c>0,(a-p+$ $1)(e-p+1)>b c$. There exists $C(x), D(x) \in C\left(\bar{\Omega}, R^{+}\right), \gamma(x), \eta(x) \in C\left(\bar{\Omega}, R^{+}\right)$such that

$$
\lim _{x \rightarrow x_{0}} \frac{a(x)}{C\left(x_{0}\right) d(x)^{r\left(x_{0}\right)}}=1, \quad \lim _{x \rightarrow x_{0}} \frac{b(x)}{D\left(x_{0}\right) d(x)^{\eta\left(x_{0}\right)}}=1,
$$

where $x_{0} \in \partial \Omega, d(x)=\operatorname{dist}(x, \partial \Omega)$. 
We must emphasize that the weight functions $a(x), b(x)$ are allowed decaying to zero on $\Omega$ with arbitrary rate, depending upon the particular point of $\partial \Omega$. The boundary condition is to be understood $u(x) \rightarrow \infty, v(x) \rightarrow \infty$ as $d(x) \rightarrow 0^{+}$. Problems like (1.1) are usually known in the literature as boundary blow-up problems, and their solutions are also named large solutions or boundary blow-up solutions.

The problem of the previous form is mathematical models occuring in studies of the $p$-Laplace system, generalized reaction-diffusion theory, non-Newtonian fluid theory $[1,2]$, non-Newtonian filtration [3], and the turbulent flow of a gas in porous medium. In the nonNewtonian fluid theory, the quantity $p$ is a characteristic of the medium. Media with $p>$ 2 are called dilatant fluids and those with $p<2$ are called pseudoplastics. If $p=2$, they are Newtonian fluids. When $p \neq 2$, the problem becomes more complicated since certain nice properties inherent to the case $p=2$ seem to be lost or at least difficult to verify. The main differences between $p=2$ and $p \neq 2$ can be founded in $[4,5]$.

When $p=2$, system (1.1) becomes

$$
\begin{aligned}
& \Delta u=a(x) u^{a} v^{b} \quad \text { in } \Omega, \\
& \Delta v=b(x) u^{c} v^{e} \quad \text { in } \Omega, \\
& u=v=+\infty \quad \text { on } \partial \Omega,
\end{aligned}
$$

for which the existence, uniqueness, and asymptotic behavior of large solutions have been investigated extensively. We list here, for example, [6-12].

This is a huge amount of literature dealing with single equation with infinite boundary conditions (see, e.g., [13-34]). This problem with more general nonlinearies and weightfunction has been discussed by many authors recently [35-39].

Problem (1.1) is considered in special case. When $p=2$, in [40], problem (1.1) was analyzed with $a(x)=1, b(x)=1$. In the same paper, some existence, uniqueness, and boundary behavior of solutions were obtained under the assumptions

$$
a(x) \sim C_{1} d(x)^{k_{1}}, \quad b(x) \sim C_{2} d(x)^{k_{2}}
$$

as $d(x) \rightarrow 0^{+}$for some positive constants $C_{1}, C_{2}$ and real numbers $k_{1}, k_{2}>-2$. This problem was later studied in [41] with general form, where

$$
\begin{aligned}
& C_{1} d(x)^{\gamma_{1}} \leq a(x) \leq C_{2} d(x)^{\gamma_{1}}, \\
& C_{1}^{\prime} d(x)^{\gamma_{1}} \leq b(x) \leq C_{2}^{\prime} d(x)^{\gamma_{1}},
\end{aligned}
$$

for $x \in \Omega, \gamma_{1}, \gamma_{2} \in \mathbf{R}^{\mathbf{N}}, C_{1}, C_{2}, C_{1}^{\prime}, C_{2}^{\prime}$ are positive constants. The author also obtained uniqueness results.

In [42], Yang extended the quasilinear elliptic system to

$$
\begin{aligned}
& \Delta_{p} u=u^{m_{1}} v^{n_{1}} \quad \text { in } \Omega, \\
& \Delta_{q} v=u^{m_{2}} v^{n_{2}} \quad \text { in } \Omega, \\
& u=v=+\infty \quad \text { on } \partial \Omega,
\end{aligned}
$$


where $m_{1}>p-1, n_{2}>q-1, m_{2}, n_{1}>0$, and $\Omega \subseteq \mathbf{R}^{\mathbf{N}}$ is a smooth bounded domain, subject to three different types of Dirichlet boundary conditions: $u=\lambda, v=\mu$ or $u=v=+\infty$ or $u=$ $+\infty, v=\mu$ on $\partial \Omega$, where $\lambda, \mu>0$. Under several hypotheses on the parameters $m_{1}, n_{1}, m_{2}, n_{2}$, the author showed the existence of positive solutions and further provided the asymptotic behavior of the solutions near $\partial \Omega$.

When $p \neq 2$, in [43], problem (1.1) was analyzed with $a(x)=1, b(x)=1$ under assumption (1.4). The author obtained the existence, uniqueness, and behavior of solutions to problem (1.1).

Very recently, Huang et al. [12] obtained existence, uniqueness, and asymptotic behavior of problem (1.1) when $p=2$, and $a(x), b(x)$ satisfy condition (1.2). Motivated by the results of the papers $[12,40,41,43]$, we consider the quasilinear elliptic system (1.1). We modify the method developed by Huang et al. [12] and extend the results to a quasilinear elliptic system (1.1) under condition (1.2).

Throughout of this paper, set

$$
\begin{gathered}
C_{1}=\min _{x \in \bar{\Omega}} C(x), \quad C_{2}=\max _{x \in \bar{\Omega}} C(x), \quad D_{1}=\min _{x \in \bar{\Omega}} D(x), \quad D_{2}=\max _{x \in \bar{\Omega}} D(x), \\
\gamma_{1}=\max _{x \in \bar{\Omega}} \gamma(x), \quad \gamma_{2}=\min _{x \in \bar{\Omega}} \gamma(x), \quad \eta_{1}=\max _{x \in \bar{\Omega}} \eta(x), \quad \eta_{2}=\min _{x \in \bar{\Omega}} \eta(x), \\
\alpha(x, y)=\frac{(p+x)(e-p+1)-(p+y) b}{(a-p+1)(e-p+1)-b c}, \quad \beta(x, y)=\frac{(p+y)(a-p+1)-(p+x) c}{(a-p+1)(e-p+1)-b c} \\
E(x, y)=\left(\frac{\left((p-1) \alpha^{p-1}(\alpha+1)\right)^{e-p+1} x^{b}}{\left((p-1) \beta^{p-1}(\beta+1)\right)^{b} y^{e-p+1}}\right)^{1 /((a-p+1)(e-p+1)-b c)} \\
F(x, y)=\left(\frac{\left((p-1) \beta^{p-1}(\beta+1)\right)^{a-p+1} y^{c}}{\left((p-1) \alpha^{p-1}(\alpha+1)\right)^{c} x^{a-p+1}}\right)^{1 /((a-p+1)(e-p+1)-b c)}
\end{gathered}
$$

$n_{x_{0}}$ stands for the outward unit normal at $x_{0} \in \partial \Omega$.

The paper is organized as follows. In Section 2 we consider some preliminaries which will be used in proof of Theorem 1.1. In Section 3 we will give the proof of the main theorem.

By modifications of the arguments in the proof of Theorem 1.1 in [12], we obtain the following main results.

Theorem 1.1. Assume that $\Omega$ is a bounded $C^{2}$ domain of $\mathbf{R}^{\mathbf{N}}, a(x), b(x) \in C^{\theta}(\Omega)$ for some $\theta \in$ $(0,1), a(x), b(x)>0$ in $\Omega$ and verify (1.2), $(a-p+1)(e-p+1)>b c, a, e>p-1, b, c>$ $0, \gamma(x), \eta(x) \in C\left(\bar{\Omega}, R^{+}\right)$and satisfy

$$
\frac{b}{e-p+1}<\frac{p+\gamma\left(x_{0}\right)}{p+\eta\left(x_{0}\right)}<\frac{a-p+1}{c} \text { for } x_{0} \in \partial \Omega \text {. }
$$


Then problem (1.1) has a solution $(u, v)$ if and only if

$$
\frac{b}{e-p+1}<\frac{p+\gamma_{1}}{p+\eta_{2}}, \quad \frac{p+\gamma_{2}}{p+\eta_{1}}<\frac{a-p+1}{c}
$$

And one has

$$
\begin{aligned}
& \lim _{x \rightarrow x_{0}} \frac{u(x)}{d(x)^{-\alpha\left(\gamma\left(x_{0}\right), \eta\left(x_{0}\right)\right)} E\left(D\left(x_{0}\right), C\left(x_{0}\right)\right)}=1, \\
& \lim _{x \rightarrow x_{0}} \frac{v(x)}{d(x)^{-\beta\left(\gamma\left(x_{0}\right), \eta\left(x_{0}\right)\right)} F\left(D\left(x_{0}\right), C\left(x_{0}\right)\right)}=1 .
\end{aligned}
$$

\section{Preliminaries}

In this section, we will introduce some propositions.

Definition 2.1. $(\underline{u}, \underline{v})$ is a subsolution of

$$
\left\{\begin{array} { l l } 
{ \Delta _ { p } u = a ( x ) u ^ { a } v ^ { b } } & { \text { in } \Omega , } \\
{ \Delta _ { p } v = b ( x ) u ^ { c } v ^ { e } } & { \text { in } \Omega , }
\end{array} \text { provided } \left\{\begin{array}{ll}
\Delta_{p} \underline{u} \geq a(x) \underline{u}^{a} \underline{v}^{b} & \text { in } \Omega, \\
\Delta_{p} \underline{v} \leq b(x) \underline{u}^{c} \underline{v}^{e} & \text { in } \Omega .
\end{array}\right.\right.
$$

A supersolution $(\bar{u}, \bar{v})$ is defined by reversing the inequalities.

Proposition 2.2. Assume that $(\underline{u}, \underline{v})$ is a subsolution and $(\bar{u}, \bar{v})$ is a supersolution of problem (1.1), with $\underline{u}=\underline{v}=\bar{u}=\bar{v}=+\infty$ on $\partial \Omega$. Then problem (1.1) has at least a solution $(u, v)$ with $\underline{u} \leq u \leq$ $\bar{u}, \underline{v} \geq v \geq \bar{v}$ in $\Omega$. In particular $u=v=+\infty$ on $\partial \Omega$.

Proposition 2.3 (see [43]). Assume that $a(x), b(x)$ satisfy (1.4), then problem (1.1) admits a positive solution $(u, v)$ with $u=v=+\infty$ on $\partial \Omega$ if and only if $k_{1}, k_{2}>-p$ and

$$
\frac{b}{e-p+1}<\frac{p+k_{1}}{p+k_{2}}<\frac{a-p+1}{c}
$$

This solution is unique and satisfies

$$
\lim _{x \rightarrow x_{0}} \frac{u(x)}{d(x)^{-\alpha\left(k_{1}, k_{2}\right)} E\left(C_{2}, C_{1}\right)}=1, \quad \lim _{x \rightarrow x_{0}} \frac{v(x)}{d(x)^{-\beta\left(k_{1}, k_{2}\right)} F\left(C_{2}, C_{1}\right)}=1
$$

for each $x_{0} \in \partial \Omega$.

Next, we are ready to study two auxiliary problems in a ball and an annuli. To this aim, for given $0<R_{1}<R$ and $x_{0} \in \mathbf{R}^{\mathbf{N}}, N \geq 1$, set

$$
B_{R}\left(x_{0}\right)=\left\{x \in \mathbf{R}^{\mathbf{N}}:\left|x-x_{0}\right|<R\right\}, \quad A_{R_{1}, R}\left(x_{0}\right)=\left\{x \in \mathbf{R}^{\mathbf{N}}: R_{1}<\left|x-x_{0}\right|<R\right\} .
$$


Proposition 2.4. Assume $\Omega=B_{R}\left(x_{0}\right),(a-p+1)(e-p+1)>b c, a, e>p-1, b, c>0, C(r), D(r) \in$ $C\left([0, R], R^{+}\right)$and $\gamma, \eta>0$ satisfy

$$
\frac{b}{e-p+1}<\frac{p+\gamma}{p+\eta}<\frac{a-p+1}{c}
$$

Then the following systems

$$
\begin{gathered}
\Delta_{p} \Phi=C(r)(R-r)^{r} \Phi^{a} \Psi^{b} \quad \text { in } B_{R}\left(x_{0}\right), \\
\Delta_{p} \Psi=D(r)(R-r)^{\eta} \Phi^{c} \Psi^{e} \quad \text { in } B_{R}\left(x_{0}\right), \\
\Phi=\Psi=+\infty \quad \text { on } \partial B_{R}\left(x_{0}\right)
\end{gathered}
$$

possess a unique radially symmetric positive solution $(\Phi(r), \Psi(r))$ satisfying

$$
\begin{aligned}
& \lim _{x \rightarrow x_{0}} \frac{\Phi(r)}{E(D(R), C(R))(R-r)^{-\alpha(r, \eta)}}=1, \\
& \lim _{x \rightarrow x_{0}} \frac{\Psi(r)}{F(D(R), C(R))(R-r)^{-\beta(r, \eta)}}=1,
\end{aligned}
$$

where $r=\left|x-x_{0}\right|$.

Proof. At first, we consider the following systems

$$
\begin{gathered}
\left(\left|\Phi^{\prime}\right|^{p-2} \Phi^{\prime}\right)^{\prime}+\frac{N-1}{r}\left(\left|\Phi^{\prime}\right|^{p-2} \Phi^{\prime}\right)=C(r)(R-r)^{r} \Phi^{a} \Psi^{b} \quad \text { in }(0, R) \\
\left(\left|\Psi^{\prime}\right|^{p-2} \Psi^{\prime}\right)^{\prime}+\frac{N-1}{r}\left(\left|\Psi^{\prime}\right|^{p-2} \Psi^{\prime}\right)=D(r)(R-r)^{\eta} \Phi^{c} \Psi^{e} \quad \text { in }(0, R) \\
\Phi(R)=\Psi(R)=+\infty, \quad \Phi^{\prime}(0)=\Psi^{\prime}(0)=0 .
\end{gathered}
$$

We will show that problem $(2.8)$ has a solution $(\Phi(r), \Psi(r))$, which provide a positive radially symmetric solution to problem (2.6). Indeed, any positive solution $(\Phi(r), \Psi(r))$ of the integral equation system

$$
\begin{gathered}
\Phi(r)=l+\int_{0}^{r}\left[t^{1-N} \int_{0}^{t} s^{N-1} C(s)(R-s)^{r} \Phi^{a} \Psi^{b} d s\right]^{1 /(p-1)} d t, \quad 0<r<R, \\
\Psi(r)=m+\int_{0}^{r}\left[t^{1-N} \int_{0}^{t} s^{N-1} D(s)(R-s)^{\eta} \Phi^{c} \Psi^{e} d s\right]^{1 /(p-1)} d t, \quad 0<r<R,
\end{gathered}
$$

provides a solution of (2.8), where $\Phi(0)=l, \Psi(0)=m, \Phi(R)=+\infty, \Psi(R)=+\infty$. 
Define $\Phi_{0}(r)=l, \Psi_{0}(r)=m$ for all $0<r<R$, let $\left\{\Phi_{k}\right\},\left\{\Psi_{k}\right\}$ be the function sequences given by

$$
\begin{gathered}
\Phi_{k}(r)=l+\int_{0}^{r}\left[t^{1-N} \int_{0}^{t} s^{N-1} C(s)(R-s)^{r} \Phi_{k-1}^{a} \Psi_{k-1}^{b} d s\right]^{1 /(p-1)} d t, \quad 0<r<R, \\
\Psi_{k}(r)=m+\int_{0}^{r}\left[t^{1-N} \int_{0}^{t} s^{N-1} D(s)(R-s)^{\eta} \Phi_{k-1}^{c} \Psi_{k-1}^{e} d s\right]^{1 /(p-1)} d t, \quad 0<r<R,
\end{gathered}
$$

subject to $\Phi_{k}(0)=l, \Psi_{k}(0)=m, \Phi_{k}(R)=\Psi_{k}(R)=k$.

We remark that $\left\{\Phi_{k}\right\},\left\{\Psi_{k}\right\}$ are nondecreasing sequences. In fact,

$$
\begin{aligned}
\Phi_{1}(r) & =l+\left(l^{a} m^{b}\right)^{1 /(p-1)} \int_{0}^{r}\left[t^{1-N} \int_{0}^{t} s^{N-1} C(s)(R-s)^{r} d s\right]^{1 /(p-1)} d t \\
& =l+\left(l^{a} m^{b}\right)^{1 /(p-1)} A(r) \geq l=\Phi_{0}(r), \\
\Psi_{1}(r) & =m+\left(l^{c} m^{e}\right)^{1 /(p-1)} \int_{0}^{r}\left[t^{1-N} \int_{0}^{t} s^{N-1} D(s)(R-s)^{\eta} d s\right]^{1 /(p-1)} d t \\
& =m+\left(l^{c} m^{e}\right)^{1 /(p-1)} B(r) \geq m=\Psi_{0}(r),
\end{aligned}
$$

where

$$
\begin{aligned}
& A(r)=\int_{0}^{r}\left[t^{1-N} \int_{0}^{t} s^{N-1} C(s)(R-s)^{r} d s\right]^{1 /(p-1)} d t \\
& B(r)=\int_{0}^{r}\left[t^{1-N} \int_{0}^{t} s^{N-1} D(s)(R-s)^{\eta} d s\right]^{1 /(p-1)} d t .
\end{aligned}
$$

Proceeding by the same manner, we conclude that

$$
l \leq \Phi_{k} \leq \Phi_{k+1}, \quad m \leq \Psi_{k} \leq \Psi_{k+1} .
$$

We now prove that $\left\{\Phi_{k}\right\},\left\{\Psi_{k}\right\}$ are bounded in $(0, R)$. To prove this, we consider

$$
\Delta_{p} \Upsilon=\left(C(r)(R-r)^{r}+D(r)(R-r)^{\eta}\right)\left(\Upsilon^{a+b}+\Upsilon^{c+e}\right)
$$

problem (2.14) has a large radially symmetric solution $\Upsilon(r)$, and

$$
\Upsilon(r)=\Upsilon(0)+\int_{0}^{r}\left[t^{1-N} \int_{0}^{t} s^{N-1}\left(C(s)(R-s)^{\gamma}+D(s)(R-s)^{\eta}\right)\left(\Upsilon^{a+b}+\Upsilon^{c+e}\right) d s\right]^{1 /(p-1)} d t
$$


where $\Upsilon(0)=l+m$. It follows that

$$
\begin{aligned}
\Phi_{1}(r) & =l+\left(l^{a} m^{b}\right)^{1 /(p-1)} \int_{0}^{r}\left[t^{1-N} \int_{0}^{t} s^{N-1} C(s)(R-s)^{\gamma} d s\right]^{1 /(p-1)} d t \\
& \leq \Upsilon(0)+\int_{0}^{r}\left[t^{1-N} \int_{0}^{t} s^{N-1}\left(C(s)(R-s)^{\gamma}+D(s)(R-s)^{\eta}\right)\left(\Upsilon^{a+b}+\Upsilon^{c+e}\right) d s\right]^{1 /(p-1)} d t \\
& =\Upsilon(r) .
\end{aligned}
$$

Similarly, we have $\Psi_{1} \leq \Upsilon(r)$.

Arguing as before, we obtain $\Phi_{k} \leq \Upsilon(r), \Psi_{k} \leq \Upsilon(r)$. Therefore, we show that $\left\{\Phi_{k}\right\},\left\{\Psi_{k}\right\}$ are nondecreasing and bounded sequences in $(0, R)$, which implies that the following limit holds

$$
(\Phi, \Psi)=\lim _{k \rightarrow \infty}\left(\Phi_{k}, \Psi_{k}\right)
$$

we deduce that $(\Phi, \Psi)$ is a positive solution of $(2.8)$. Then $(\Phi(x), \Psi(x))=(\Phi(r), \Psi(r))$ is a positive radially symmetric solution to problem (2.6) and

$$
\Phi(R)=\lim _{r \rightarrow R} \Phi(r)=\infty, \quad \Psi(R)=\lim _{r \rightarrow R} \Psi(r)=\infty
$$

Secondly, it is clear that

$$
C_{1}(R-r)^{\gamma} \leq C(r)(R-r)^{\gamma} \leq C_{2}(R-r)^{\gamma}, \quad D_{1}(R-r)^{\eta} \leq D(r)(R-r)^{\eta} \leq D_{2}(R-r)^{\eta} .
$$

By (2.5) and Proposition 2.3, we have

$$
\begin{aligned}
& E\left(D_{1}, C_{2}\right) \leq \lim _{r \rightarrow R} \frac{\Phi(r)}{(R-r)^{-\alpha(\gamma, \eta)}} \leq E\left(D_{2}, C_{1}\right), \\
& F\left(D_{2}, C_{1}\right) \leq \lim _{r \rightarrow R} \frac{\Psi(r)}{(R-r)^{-\beta(\gamma, \eta)}} \leq F\left(D_{1}, C_{2}\right) .
\end{aligned}
$$

Denote by

$$
l=\lim _{r \rightarrow R} \frac{\Phi(r)}{(R-r)^{-\alpha(\gamma, \eta)}}, \quad k=\lim _{r \rightarrow R} \frac{\Psi(r)}{(R-r)^{-\beta(\gamma, \eta)}} .
$$


By using $\gamma+p=(a-p+1) \alpha(\gamma, \eta)+b \beta(\gamma, \eta), \eta+p=(e-p+1) \beta(\gamma, \eta)+c \alpha(\gamma, \eta)$ and L'Hôpital rule, we obtain

$$
\begin{aligned}
l= & \lim _{r \rightarrow R} \frac{\Phi(0)+\int_{0}^{r}\left[t^{1-N} \int_{0}^{t} s^{N-1} C(s)(R-s)^{r} \Phi^{a} \Psi^{b} d s\right]^{1 /(p-1)} d t}{(R-r)^{-\alpha}} \\
= & \lim _{r \rightarrow R} \frac{\left[r^{1-N} \int_{0}^{r} t^{N-1} C(t)(R-t)^{r} \Phi^{a} \Psi^{b} d t\right]^{1 /(p-1)}}{\alpha(R-r)^{-\alpha-1}} \\
= & {\left[\lim _{r \rightarrow R} \frac{r^{1-N} \int_{0}^{r} t^{N-1} C(t)(R-t)^{r} \Phi^{a} \Psi^{b} d t}{\left.\alpha(R-r)^{-(\alpha+1)(p-1)}\right]^{1 /(p-1)}}\right.} \\
= & {\left[\lim _{r \rightarrow R} \frac{(1-N) r^{-N} \int_{0}^{r} t^{N-1} C(t)(R-t)^{r} \Phi^{a} \Psi^{b} d t+C(r)(R-r)^{\gamma} \Phi^{a} \Psi^{b}}{\alpha(\alpha+1)(p-1)(R-r)^{-\alpha p+\alpha-p}}\right]^{1 /(p-1)} } \\
= & {\left[\frac{C(R)}{\alpha(\alpha+1)(p-1)} \lim _{r \rightarrow R}(R-r)^{a \alpha+b \beta} \Phi^{a} \Psi^{b}\right.} \\
& \left.+\frac{1-N}{\alpha(\alpha+1)(p-1)} \lim _{r \rightarrow R} \frac{r^{-N} \int_{0}^{r} t^{N-1} C(t)(R-t)^{r} \Phi^{a} \Psi^{b} d t}{(R-r)^{-\alpha p+\alpha-p}}\right]^{1 /(p-1)} \\
= & {\left[\frac{C(R)}{\alpha(\alpha+1)(p-1)} l^{a} k^{b}+\frac{1-N}{\alpha(\alpha+1)(p-1)} \lim _{r \rightarrow R} \frac{r^{-N} \int_{0}^{r} t^{N-1} C(t)(R-t)^{\gamma} \Phi^{a} \Psi^{b} d t}{(R-r)^{-\alpha p+\alpha-p}}\right]^{1 /(p-1)} . }
\end{aligned} .
$$

We note that

$$
\begin{aligned}
0 & \leq \lim _{r \rightarrow R} \frac{r^{-N} \int_{0}^{r} t^{N-1} C(t)(R-t)^{\gamma} \Phi^{a} \Psi^{b} d t}{(R-r)^{-\alpha p+\alpha-p}} \\
& \leq \lim _{r \rightarrow R} \frac{r^{-1} \int_{0}^{r} C(t)(R-t)^{r} \Phi^{a} \Psi^{b} d t}{(R-r)^{-\alpha p+\alpha-p}} \\
& =\lim _{r \rightarrow R} \frac{C(r)(R-r)^{\gamma} \Phi^{a} \Psi^{b} d t}{R(-\alpha p+\alpha-p)(R-r)^{-\alpha p+\alpha-p+1}} \\
& =\frac{C(R)}{R(-\alpha p+\alpha-p)} \lim _{r \rightarrow R}(R-r)^{r+\alpha p-\alpha+p+1} \Phi^{a} \Psi^{b} \\
& =\frac{C(R)}{R(-\alpha p+\alpha-p)} l^{a} k^{b} \lim _{r \rightarrow R}(R-r)=0 .
\end{aligned}
$$

This implies that

$$
l^{p-1}=\frac{C(R)}{\alpha(\alpha+1)(p-1)} l^{a} k^{b} .
$$


Similarly, we obtain

$$
k^{p-1}=\frac{D(R)}{\beta(\beta+1)(p-1)} l^{c} k^{e}
$$

Since

$$
\begin{aligned}
& \frac{\alpha^{p-1}(\alpha+1)(p-1)}{C(R)}=E(D(R), C(R))^{a-p+1} F(D(R), C(R))^{b} \\
& \frac{\beta^{p-1}(\beta+1)(p-1)}{D(R)}=E(D(R), C(R))^{c} F(D(R), C(R))^{e-p+1} .
\end{aligned}
$$

If $0<\alpha<1,1<p \leq 2$, then

$$
E^{a-p+1} F^{b}=\frac{\alpha^{p-1}(\alpha+1)(p-1)}{C(R)} \geq \frac{\alpha(\alpha+1)(p-1)}{C(R)}=l^{a-p+1} k^{b}
$$

therefore, we get $E \geq l, F \geq k$. If $0<\alpha<1, p>2$, we get $E \leq l, F \leq k$. So, when $0<\alpha<1$, we get $E=l, F=k$.

Similarly, when $\alpha \geq 1$, we also get $E=l, F=k$.

By (2.24) and (2.25), we conclude that $l=E(D(R), C(R)), k=F(D(R), C(R))$, this completes the proof.

Proposition 2.5. Assume $(a-p+1)(e-p+1)>b c, a, e>p-1, b, c>0, \gamma>0, \eta>0$, and

$$
\frac{b}{e-p+1}<\frac{p+\gamma}{p+\eta}<\frac{a-p+1}{c}
$$

$D(r), C(r) \in C\left(\left[R_{1}, R\right], R^{+}\right)$are the reflection around $R_{0}=\left(R_{1}+R\right) / 2$ of some functions $\bar{C}(r), \bar{D}(r) \in C\left(\left[R_{0}, R\right], R^{+}\right)$. Then the following system

$$
\begin{gathered}
\Delta_{p} \Phi=C(r) d(x)^{r} \Phi^{a} \Psi^{b} \quad \text { in } A_{R_{1}, R}\left(x_{0}\right), \\
\Delta_{p} \Psi=D(r) d(x)^{\eta} \Phi^{c} \Psi^{e} \quad \text { in } A_{R_{1}, R}\left(x_{0}\right), \\
\Phi=\Psi=+\infty \quad \text { on } \partial A_{R_{1}, R}\left(x_{0}\right)
\end{gathered}
$$

has a unique radially symmetric positive solution $(\Phi(r), \Psi(r))$ such that

$$
\begin{aligned}
& \lim _{d(x) \rightarrow 0} \frac{\Phi(r)}{E(D(R), C(R)) d(x)^{-\alpha(\gamma, \eta)}}=1, \\
& \lim _{d(x) \rightarrow 0} \frac{\Psi(r)}{F(D(R), C(R)) d(x)^{-\beta(\gamma, \eta)}}=1,
\end{aligned}
$$


where

$$
d(x)=d\left(x, \partial A_{R_{1}, R}\left(x_{0}\right)\right)= \begin{cases}R-\left|x-x_{0}\right|, & \text { if } R_{0} \leq\left|x-x_{0}\right| \leq R, \\ \left|x-x_{0}\right|-R_{1}, & \text { if } R_{1} \leq\left|x-x_{0}\right| \leq R_{0} .\end{cases}
$$

Proof. The proof is similarl to the proof of Proposition 2.4, so we omit it here.

\section{Proof of Theorem 1.1}

We are now ready to prove Theorem 1.1, whose proof will be split into the following several lemmas.

Lemma 3.1. Assume $(a-p+1)(e-p+1)>b c, a, e>p-1, b, c>0, C(x), D(x) \in$ $C(\bar{\Omega}), a(x), b(x)>0$ in $\Omega$ and (1.2) holds, $\gamma(x), \eta(x)>0$ and satisfy

$$
\frac{b}{e-p+1}<\frac{p+\gamma\left(x_{0}\right)}{p+\eta\left(x_{0}\right)}<\frac{a-p+1}{c}
$$

for each $x_{0} \in \partial \Omega$, then problem (1.1) has a solution $(u, v)$ if

$$
\frac{b}{e-p+1}<\frac{p+\gamma_{1}}{p+\eta_{2}}, \quad \frac{p+\gamma_{2}}{p+\eta_{1}}<\frac{a-p+1}{c}
$$

Proof. By (3.2) and Proposition 2.3, the following system

$$
\begin{gathered}
\Delta_{p} \Phi=C_{1} d(x)^{\gamma_{1}} \Phi^{a} \Psi^{b} \quad \text { in } \Omega, \\
\Delta_{p} \Psi=D_{2} d(x)^{\eta_{2}} \Phi^{c} \Psi^{e} \quad \text { in } \Omega, \\
\Phi=\Psi=+\infty \quad \text { on } \partial \Omega
\end{gathered}
$$

possesses a positive solution $\left(u_{1}, v_{1}\right)$.

Next we will show that

$$
(\bar{u}, \bar{v})=\left(\left(\frac{m+n}{(m-n)^{b /(a-p+1)}}\right)^{1 /(p-1)} u_{1},\left(\frac{m-n}{(m+n)^{c /(e-p+1)}}\right)^{1 /(p-1)} v_{1}\right)
$$

is a supersolution of (1.1), if $m$ is sufficiently large and $0<m-n<1$, where $m, n \in R^{+}$and $m>n$. In fact, by

$$
\gamma_{1}+p=(a-p+1) \alpha\left(\gamma_{1}, \eta_{2}\right)+b \beta\left(\gamma_{1}, \eta_{2}\right), \quad \eta_{2}+p=(e-p+1) \beta\left(\gamma_{1}, \eta_{2}\right)+c \alpha\left(\gamma_{1}, \eta_{2}\right) .
$$


We have $(\bar{u}, \bar{v})$ is a supersolution of (1.1) provided

$$
\begin{gathered}
C_{1} d(x)^{\gamma_{1}} \leq a(x)(m+n)^{((e-p+1)(a-p+1)-b c) /(e-p+1)(p-1),} \\
D_{2} d(x) \geq b(x)(m-n)^{((a-p+1)(e-p+1)-b c) /(a-p+1)(p-1)} .
\end{gathered}
$$

Since $a(x), b(x) \in C(\bar{\Omega})$, choosing $m$ is large enough, and $m-n>0$ is sufficiently small, we can prove that

$$
(\underline{u}, \underline{v})=\left(\left(\frac{m-n}{(m+n)^{b /(a-p+1)}}\right)^{1 /(p-1)} u_{2},\left(\frac{m+n}{(m-n)^{c /(e-p+1)}}\right)^{1 /(p-1)} v_{2}\right)
$$

is a subsolution of $(1.1)$, where $\left(u_{2}, v_{2}\right)$ is a solution of the following problem:

$$
\begin{gathered}
\Delta_{p} \Phi=C_{2} d(x)^{\gamma_{2}} \Phi^{a} \Psi^{b} \quad \text { in } \Omega, \\
\Delta_{p} \Psi=D_{1} d(x)^{\eta_{1}} \Phi^{c} \Psi^{e} \quad \text { in } \Omega, \\
\Phi=\Psi=+\infty \quad \text { on } \partial \Omega .
\end{gathered}
$$

Then by Proposition 2.2, problem (1.1) has a solution.

Lemma 3.2. Assume that problem (1.1) has a solution $(u, v)$, then (1.9) holds.

Proof. In fact, if (1.9) does not hold, it will lead to a contradiction. From Lemma 3.1, we find that if $m$ is large enough and $m-n>0$ is sufficiently small, we have

$$
u \leq \bar{u}=\left(\frac{m+n}{(m-n)^{b /(a-p+1)}}\right)^{1 /(p-1)} u_{1}, \quad v \leq \underline{v}=\left(\frac{m+n}{(m-n)^{c /(e-p+1)}}\right)^{1 /(p-1)} v_{2} .
$$

On the other hand, by (2.3), there exists $\varepsilon>0$ such that for $x \in \Omega_{\varepsilon}=\{x \in \Omega: d(x, \partial \Omega) \leq \varepsilon\}$, we get

$$
u \leq\left(\frac{m+n}{(m-n)^{b /(a-p+1)}}\right)^{1 /(p-1)} u_{1} \leq\left(\frac{m+n}{(m-n)^{b /(a-p+1)}}\right)^{1 /(p-1)} E\left(D_{2}, C_{1}\right) d(x)^{-\alpha\left(\gamma_{1}, \eta_{2}\right)}
$$

Thus, if

$$
\frac{b}{e-p+1} \geq \frac{p+\gamma_{1}}{p+\eta_{2}}
$$


by the definition of $\alpha\left(\gamma_{1}, \eta_{2}\right)$, we obtain $\alpha\left(\gamma_{1}, \eta_{2}\right) \leq 0$. By (3.10), it implies that $u$ is bounded for $x \in \Omega_{\varepsilon}$, which is impossible since $u(x)=+\infty$ as $d(x)=\operatorname{dist}(x, \partial \Omega) \rightarrow 0^{+}$. If

$$
\frac{p+\gamma_{2}}{p+\eta_{2}} \geq \frac{a-p+1}{c}
$$

it is similarly proved that $v$ is bounded near $\partial \Omega$, which is also a contradiction. The proof of Lemma 3.2 is complete.

Lemma 3.3. Let $(u, v)$ be a positive solution of (1.1), then (1.10) and (1.11) hold.

Proof. Fix $\tau \in(0,1)$, by $(1.2)$, there exits $\sigma \in(0,1)$ such that, if $d\left(x, x_{0}\right)<\sigma$,

$$
a(x) \geq(1-\tau) C\left(x_{0}\right) d(x)^{\gamma\left(x_{0}\right)}, \quad b(x) \leq(1+\tau) D\left(x_{0}\right) d(x)^{\eta\left(x_{0}\right)},
$$

where $x_{0} \in \partial \Omega$. For a fixed $x_{0} \in \partial \Omega$, set

$$
\Sigma=\bar{B}_{\sigma / 2} \bigcap \partial \Omega
$$

and choose $R>0$ small enough such that

$$
K=\bigcup_{y \in \Sigma} \bar{B}_{R}\left(y-R n_{y}\right) \subset B_{\sigma}\left(x_{0}\right) \bigcap \Omega,
$$

where $n_{y}$ stands for the outward unit normal at $y \in \partial \Omega$.

For $x \in B_{\sigma}\left(x_{0}\right) \cap \Omega$, we get

$$
a(x) \geq(1-\tau) C\left(x_{0}\right) d(x)^{\gamma\left(x_{0}\right)}, \quad b(x) \leq(1+\tau) D\left(x_{0}\right) d(x)^{\eta\left(x_{0}\right)} .
$$

Since $\Omega$ is of $C^{2}$ bounded domain, there exit $R>0$ and $\sigma_{0}>0$ such that

$$
B_{R}\left(x_{0}-(R+\sigma) n_{x_{0}}\right) \subset \Omega, \quad B_{R}\left(x_{0}-R n_{x_{0}}\right) \bigcap \partial \Omega=\left\{x_{0}\right\},
$$

for each $\sigma \in\left(0, \sigma_{0}\right)$.

Let $\left(u_{B, \sigma}, v_{B, \sigma}\right)$ be any positive radially symmetric solution to the following system:

$$
\begin{gathered}
\Delta_{p} u=(1-\tau) C\left(x_{0}\right)\left(R-\left|x-x_{0}\right|\right)^{\gamma\left(x_{0}\right)} u^{a} v^{b} \quad \text { in } B_{R}\left(x_{0}-(R+\sigma) n_{x_{0}}\right), \\
\Delta_{p} v=(1+\tau) D\left(x_{0}\right)\left(R-\left|x-x_{0}\right|\right)^{\eta\left(x_{0}\right)} u^{c} v^{e} \quad \text { in } B_{R}\left(x_{0}-(R+\sigma) n_{x_{0}}\right), \\
u=v=+\infty \quad \text { on } \partial B_{R}\left(x_{0}-(R+\sigma) n_{x_{0}}\right) .
\end{gathered}
$$

It is easy to see that $\left(\underline{u}_{\sigma}, \underline{v}_{\sigma}\right)=\left.(u, v)\right|_{B_{R}\left(x_{0}-(R+\sigma) n_{x_{0}}\right)}$ is a positive smooth subsolution of (3.18), where $(u, v)$ is a positive solution of (1.1). 
Then we get

$$
\underline{u}_{\sigma}=\left.u\right|_{B_{R}\left(x_{0}-(R+\sigma) n_{x_{0}}\right)} \leq u_{B, \sigma}, \quad \underline{v}_{\sigma}=\left.v\right|_{B_{R}\left(x_{0}-(R+\sigma) n_{x_{0}}\right)} \geq v_{B, \sigma}
$$

Let $\left(u_{B}, v_{B}\right)$ be any positive solution to the following system:

$$
\begin{gathered}
\Delta_{p} u=(1-\tau) C\left(x_{0}\right)\left(R-\left|x-x_{0}\right|\right)^{\gamma\left(x_{0}\right)} u^{a} v^{b} \quad \text { in } B_{R}\left(x_{0}-R n_{x_{0}}\right), \\
\Delta_{p} v=(1+\tau) D\left(x_{0}\right)\left(R-\left|x-x_{0}\right|\right)^{\eta\left(x_{0}\right)} u^{c} v^{e} \quad \text { in } B_{R}\left(x_{0}-R n_{x_{0}}\right), \\
u=v=+\infty \quad \text { on } \partial B_{R}\left(x_{0}-R n_{x_{0}}\right) .
\end{gathered}
$$

By Proposition 2.3, $\left(u_{B}, v_{B}\right)$ satisfies

$$
\begin{aligned}
& \lim _{r \rightarrow R} \frac{u_{B}}{E\left((1+\tau) D\left(x_{0}\right),(1-\tau) C\left(x_{0}\right)\right)(R-r)^{-\alpha\left(r\left(x_{0}\right), \eta\left(x_{0}\right)\right)}}=1, \\
& \lim _{r \rightarrow R} \frac{v_{B}}{F\left((1+\tau) D\left(x_{0}\right),(1-\tau) C\left(x_{0}\right)\right)(R-r)^{-\beta\left(r\left(x_{0}\right), \eta\left(x_{0}\right)\right)}}=1,
\end{aligned}
$$

where $r=\left|x-x_{0}\right|$.

Taking into account that, for $x \in B_{R}\left(x_{0}-(R+\sigma) n_{x_{0}}\right)$,

$$
u_{B, \sigma}(x)=u_{B}\left(x+\sigma n_{x_{0}}\right), \quad v_{B, \sigma}(x)=v_{B}\left(x+\sigma n_{x_{0}}\right),
$$

by (3.19), for each $x \in B_{R}\left(x_{0}-(R+\sigma) n_{x_{0}}\right)$ and $\sigma \in\left(0, \sigma_{0}\right)$, we have

$$
u(x) \leq u_{B}\left(x+\sigma n_{x_{0}}\right), \quad v(x) \geq v_{B}\left(x+\sigma n_{x_{0}}\right) .
$$

Let $\sigma \rightarrow 0$, we have

$$
u(x) \leq u_{B}(x), \quad v(x) \geq v_{B}(x) .
$$

It follows immediately from (3.21), (3.22) that

$$
\begin{aligned}
& \lim _{r \rightarrow R} \frac{u}{E(R-r)^{-\alpha}} \leq \lim _{r \rightarrow R} \frac{u_{B}}{E(R-r)^{-\alpha}}=1, \\
& \lim _{r \rightarrow R} \frac{v}{F(R-r)^{-\beta}} \geq \lim _{r \rightarrow R} \frac{v_{B}}{F(R-r)^{-\beta}}=1,
\end{aligned}
$$

where $E=E((1+\tau) D(R),(1-\tau) C(R)), F=F((1+\tau) D(R),(1-\tau) C(R))$.

We next have to prove the inverse inequalities. Similarly, there exits $R>R_{1}>0$ and $\sigma_{0}>0$ such that $\Omega \subset \bigcap_{0<\sigma<\sigma_{0}} A_{R_{1}, R}\left(x_{0}+(R+\sigma) n_{x_{0}}\right)$ and $A_{R_{0}, R}\left(x_{0}+R_{1} n_{x_{0}}\right) \cap \partial \Omega=\left\{x_{0}\right\}$. 
Fix a sufficiently small $\tau$, there exit radially symmetric functions $\bar{a}: A_{R_{1}, R}\left(x_{0}+R_{1} n_{x_{0}}\right) \rightarrow R^{+}$ and $\underline{b}: A_{R_{1}, R}\left(x_{0}+R_{1} n_{x_{0}}\right) \rightarrow R^{+}$such that $\bar{a} \geq a, \underline{b} \leq b$ in $\Omega$, and

$$
\max _{\bar{A}_{R_{1}, R\left(x_{0}+R_{1} n x_{0}\right)}} \bar{a} \leq \max _{\Omega} a+1, \quad \max _{\Omega} b+1 \leq \max _{\bar{A}_{R_{1}, R\left(x_{0}+R_{1} n x_{0}\right)}} \underline{b}
$$

and for each $x \in A_{R_{1}, R}\left(x_{0}+R_{1} n x_{0}\right)$

$$
\begin{aligned}
& \bar{a}(x)=a_{1}\left(\left|x-x_{0}-R_{1} n_{x_{0}}\right|\right)\left[d\left(x, \partial A_{R_{1}, R\left(x_{0}+R_{1} n_{x_{0}}\right)}\right)\right]^{\gamma\left(x_{0}\right)}, \\
& \underline{b}(x)=b_{1}\left(\left|x-x_{0}-R_{1} n_{x_{0}}\right|\right)\left[d\left(x, \partial A_{R_{1}, R\left(x_{0}+R_{1} n_{x_{0}}\right)}\right)\right]^{\eta\left(x_{0}\right)},
\end{aligned}
$$

where $a_{1}, b_{1} \in C\left(\left[R_{1}, R\right], R^{+}\right)$, satisfing

$$
a_{1}\left(R_{1}\right)=C\left(x_{0}\right)+\tau, \quad b_{1}\left(R_{1}\right)=D\left(x_{0}\right)-\tau .
$$

We now consider the system

$$
\begin{gathered}
\Delta_{p} u=\bar{a}(x) u^{a} v^{b} \quad \text { in } A_{R_{1}, R}\left(x_{0}+R_{1} n_{x_{0}}\right), \\
\Delta_{p} v=\underline{b}(x) u^{c} v^{e} \quad \text { in } A_{R_{1}, R}\left(x_{0}+R_{1} n_{x_{0}}\right), \\
u=v=+\infty \quad \text { on } \partial A_{R_{1}, R}\left(x_{0}+R_{1} n_{x_{0}}\right) .
\end{gathered}
$$

By Proposition 2.5, problem (3.31) possesses a solution $\left(u_{A}, v_{A}\right)$.

But for the system

$$
\begin{aligned}
& \Delta_{p} u=\bar{a}(x) u^{a} v^{b} \quad \text { in } A_{R_{1}, R}\left(x_{0}+\left(R_{1}+\sigma\right) n_{x_{0}}\right) \\
& \Delta_{p} v=\underline{b}(x) u^{c} v^{e} \quad \text { in } A_{R_{1}, R}\left(x_{0}+\left(R_{1}+\sigma\right) n_{x_{0}}\right) \\
& u=v=+\infty \quad \text { on } \partial A_{R_{1}, R}\left(x_{0}+\left(R_{1}+\sigma\right) n_{x_{0}}\right)
\end{aligned}
$$

it has a solution $\left(u_{A, \sigma}, v_{A, \sigma}\right)$, and for each $x \in A_{R_{1}, R}\left(x_{0}+\left(R_{1}+\sigma\right) n_{x_{0}}\right)$, we have

$$
\left(u_{A, \sigma}(x), v_{A, \sigma}(x)\right)=\left(u_{A}\left(x-\sigma n_{x_{0}}\right), v_{A}\left(x-\sigma n_{x_{0}}\right)\right) .
$$


It is also clear that $\left(\underline{u}_{A}(x), \underline{v}_{A}(x)\right)=\left.\left(u_{A, \sigma}(x), v_{A, \sigma}(x)\right)\right|_{\Omega}$ is a subsolution of problem (1.1). Thus for each $x \in A_{R_{1}, R}\left(x_{0}+\left(R_{1}+\sigma\right) n_{x_{0}}\right)$, we get $u_{A}\left(x-\sigma n_{x_{0}}\right) \leq u(x), v_{A}\left(x-\sigma n_{x_{0}}\right) \geq v(x)$. Let $\sigma \rightarrow 0$, we have $\underline{u}_{A}(x) \leq u(x), \underline{v}_{A}(x) \geq v(x)$. Thus for $x \in K$, we get

$$
\begin{aligned}
1 & =\lim _{|x| \rightarrow R} \frac{\underline{u}_{A}(x)}{E(\bar{a}(x), \underline{b}(x))(R-|x|)^{-\alpha\left(\gamma\left(x_{0}\right), \eta\left(x_{0}\right)\right)}} \\
& \leq \lim _{d(x) \rightarrow 0} \frac{u(x)}{E(\bar{a}(x), \underline{b}(x))(R-|x|)^{-\alpha\left(\gamma\left(x_{0}\right), \eta\left(x_{0}\right)\right)}}, \\
1 & =\lim _{|x| \rightarrow R} \frac{\underline{v}_{A}(x)}{F(\bar{a}(x), \underline{b}(x))(R-|x|)^{-\beta\left(\gamma\left(x_{0}\right), \eta\left(x_{0}\right)\right)}} \\
& \geq \lim _{d(x) \rightarrow 0} \frac{v(x)}{F(\bar{a}(x), \underline{b}(x))(R-|x|)^{-\beta\left(\gamma\left(x_{0}\right), \eta\left(x_{0}\right)\right)}}
\end{aligned}
$$

but we have $\lim _{\tau \rightarrow 0} K=\left\{x_{0}\right\}$. Therefore, by (3.26), (3.27), (3.34), and (3.35), we finish (1.10) and (1.11). The proof of Lemma 3.3 is complete. From Lemma 3.1 to Lemma 3.3, we finish the proof of Theorem 1.1.

\section{Acknowledgments}

This paper was supported by the National Natural Science Foundation of China (Grant no. 10871060) by the Natural Science Foundation of the Jiangsu Higher Education Institutions of China (Grant no. 8KJB110005).

\section{References}

[1] G. Astrita and G. Marrucci, Principles of Non-Newtonian Fluid Mechanics, McGraw-Hill, New York, NY, USA, 1974.

[2] L. K. Martinson and K. B. Pavlov, "Unsteady shear flows of a conducting fluid with a rheological power law," Magnitnaya Gidrodinamika, vol. 2, pp. 50-58, 1971.

[3] A. S. Kalashnikov, "On a nonlinaer equation appearing in the theory of non-stationary filtration," Trudy Seminara imeni I. G. Petrovskogo, vol. 4, pp. 137-146, 1978.

[4] Z. M. Guo, "Some existence and multiplicity results for a class of quasilinear elliptic eigenvalue problems," Nonlinear Analysis: Theory, Methods E Applications, vol. 18, no. 10, pp. 957-971, 1992.

[5] Z. M. Guo and J. R. L. Webb, “Uniqueness of positive solutions for quasilinear elliptic equations when a parameter is large," Proceedings of the Royal Society of Edinburgh: Section A, vol. 124, no. 1, pp. 189-198, 1994.

[6] Y. Du, "Effects of a degeneracy in the competition model. Part II. Perturbation and dynamical behaviour," Journal of Differential Equations, vol. 181, no. 1, pp. 133-164, 2002.

[7] Y. Du, "Effects of a degeneracy in the competition model. Part I. Classical and generalized steady-state solutions," Journal of Differential Equations, vol. 181, no. 1, pp. 92-132, 2002.

[8] E. N. Dancer and Y. Du, "Effects of certain degeneracies in the predator-prey model," SIAM Journal on Mathematical Analysis, vol. 34, no. 2, pp. 292-314, 2002.

[9] J. García-Melián, R. Letelier-Albornoz, and J. Sabina de Lis, "The solvability of an elliptic system under a singular boundary condition," Proceedings of the Royal Society of Edinburgh: Section A, vol. 136, no. 3, pp. 509-546, 2006.

[10] J. López-Gómez, "Coexistence and meta-coexistence for competing species," Houston Journal of Mathematics, vol. 29, no. 2, pp. 483-536, 2003. 
[11] J. García-Melián, A. Suárez, and J. Sabina de Lis, "Existence and uniqueness of positive large solutions to some cooperative elliptic systems," Advanced Nonlinear Studies, vol. 3, pp. 193-206, 2003.

[12] S. Huang, Q. Tian, and C. Mu, "Large solutions of elliptic system of competitive type: existence uniqueness and asymptotic behavior," Nonlinear Analysis: Theory, Methods \& Applications, vol. 71, pp. 4544-4552, 2009.

[13] L. Bieberbach, “ $\Delta u=e^{u}$ und die automorphen Funktionen," Mathematische Annalen, vol. 77, no. 2, pp. 173-212, 1916.

[14] C. Bandle and M. Marcus, "Sur les solutions maximales de problèmes elliptiques nonlinèaires: bornes isopèrimètriques et comportement asymptotique," Comptes Rendus de l'Acadèmie des Sciences. Sèrie I. Mathèmatique, vol. 311, no. 2, pp. 91-93, 1990.

[15] C. Bandle and M. Marcus, "Large solutions of semilinear elliptic equations: existence, uniqueness and asymptotic behaviour," Journal d'Analyse Mathématique, vol. 58, pp. 9-24, 1992.

[16] C. Bandle and M. Essèn, "On the solutions of quasilinear elliptic problems with boundary blow-up," in Partial Differential Equations of Elliptic Type, vol. 35 of Symposia Mathematica, pp. 93-111, Cambridge University Press, Cambridge, UK, 1994.

[17] C. Bandle and M. Marcus, "On second-order effects in the boundary behaviour of large solutions of semilinear elliptic problems," Differential and Integral Equations, vol. 11, no. 1, pp. 23-34, 1998.

[18] M. Chuaqui, C. Cortázar, M. Elgueta, C. Flores, R. Letelier, and J. García-Melián, “On an elliptic problem with boundary blow-up and a singular weight: the radial case," Proceedings of the Royal Society of Edinburgh: Section A, vol. 133, no. 6, pp. 1283-1297, 2003.

[19] M. Chuaqui, C. Cortázar, and J. Garcia-Melián, "Uniqueness and boundary behavior of large solutions to elliptic problems with singular weights," Communications on Pure and Applied Analysis, vol. 3, no. 4, pp. 653-662, 2004.

[20] M. del Pino and R. Letelier, "The influence of domain geometry in boundary blow-up elliptic problems," Nonlinear Analysis: Theory, Methods E Applications, vol. 48, no. 6, pp. 897-904, 2002.

[21] G. Díaz and R. Letelier, "Explosive solutions of quasilinear elliptic equations: existence and uniqueness," Nonlinear Analysis: Theory, Methods E Applications, vol. 20, no. 2, pp. 97-125, 1993.

[22] Y. Du and Q. Huang, "Blow-up solutions for a class of semilinear elliptic and parabolic equations," SIAM Journal on Mathematical Analysis, vol. 31, no. 1, pp. 1-18, 1999.

[23] J. García-Melián, "A remark on the existence of large solutions via sub and supersolutions," Electronic Journal of Differential Equations, vol. 2003, pp. 1-4, 2003.

[24] J. García-Melián, R. Letelier-Albornoz, and J. Sabina de Lis, “Uniqueness and asymptotic behaviour for solutions of semilinear problems with boundary blow-up," Proceedings of the American Mathematical Society, vol. 129, no. 12, pp. 3593-3602, 2001.

[25] J. B. Keller, "On solution of $\delta u=f(u)$," Communications on Pure E Applied Mathematics, vol. 10, pp. 503-510, 1957.

[26] C. Loewner and L. Nirenberg, "Partial differential equations invariant under conformal or projective transformations," in Contributions to Analysis (A Collection of Papers Dedicated to Lipman Bers), pp. 245272, Academic Press, New York, NY, USA, 1974.

[27] A. C. Lazer and P. J. McKenna, "On a problem of Bieberbach and Rademacher," Nonlinear Analysis: Theory, Methods \& Applications, vol. 21, no. 5, pp. 327-335, 1993.

[28] A. C. Lazer and P. J. McKenna, "Asymptotic behavior of solutions of boundary blowup problems," Differential and Integral Equations, vol. 7, no. 3-4, pp. 1001-1019, 1994.

[29] V. A. Kondrat'ev and V. A. Nikishkin, "On the asymptotic behavior near the boundary of the solution of a singular boundary value problem for a semilinear elliptic equation," Differential Equations, vol. 26, no. 3, pp. 465-468, 1990.

[30] M. Marcus and L. Véron, “Uniqueness and asymptotic behavior of solutions with boundary blow-up for a class of nonlinear elliptic equations," Annales de l'Institut Henri Poincaré. Analyse Non Linéaire, vol. 14, no. 2, pp. 237-274, 1997.

[31] A. Mohammed, G. Porcu, and G. Porru, "Large solutions to some non-linear O.D.E. with singular coefficients," Nonlinear Analysis: Theory, Methods \& Applications, vol. 47, no. 1, pp. 513-524, 2001.

[32] R. Osserman, “On the inequality $\Delta u \geq f(u)$," Pacific Journal of Mathematics, vol. 7, pp. 1641-1647, 1957.

[33] L. Véron, "Semilinear elliptic equations with uniform blow-up on the boundary," Journal d'Analyse Mathématique, vol. 59, pp. 231-250, 1992.

[34] Z. Zhang, "A remark on the existence of explosive solutions for a class of semilinear elliptic equations," Nonlinear Analysis: Theory, Methods E Applications, vol. 41, no. 1-2, pp. 143-148, 2000. 
[35] Z. D. Yang, B. Xu, and M. Wu, "Existence of positive boundary blow-up solutions for quasilinear elliptic equations via sub and supersolutions," Applied Mathematics and Computation, vol. 188, no. 1, pp. 492-498, 2007.

[36] Z. D. Yang, "Existence of explosive positive solutions of quasilinear elliptic equations," Applied Mathematics and Computation, vol. 177, no. 2, pp. 581-588, 2006.

[37] Z. D. Yang and Q. Lu, "Existence of entire explosive positive radial solutions of sublinear elliptic systems," Communications in Nonlinear Science \& Numerical Simulation, vol. 6, no. 2, pp. 88-92, 2001.

[38] Z. D. Yang, "Existence of entire explosive positive radial solutions of quasilinear elliptic systems," International Journal of Mathematics and Mathematical Sciences, no. 46, pp. 2907-2927, 2003.

[39] Z. D. Yang and H. Yang, "Existence of positive entire solutions for quasilinear elliptic systems," Annals of Differential Equations, vol. 18, no. 4, pp. 417-424, 2002.

[40] J. García-Melián and J. D. Rossi, "Boundary blow-up solutions to elliptic systems of competitive type," Journal of Differential Equations, vol. 206, no. 1, pp. 156-181, 2004.

[41] J. García-Melián, "A remark on uniqueness of large solutions for elliptic systems of competitive type," Journal of Mathematical Analysis and Applications, vol. 331, no. 1, pp. 608-616, 2007.

[42] Z. D. Yang and M. Z. Wu, "Existence of boundary blow-up solutions for a class of quasilinear elliptic systems for the subcritical case," Communications on Pure and Applied Analysis, vol. 6, no. 2, pp. 531$540,2007$.

[43] J. García-Melián, “Large solutions for an elliptic system of quasilinear equations," Journal of Differential Equations, vol. 245, no. 12, pp. 3735-3752, 2008. 


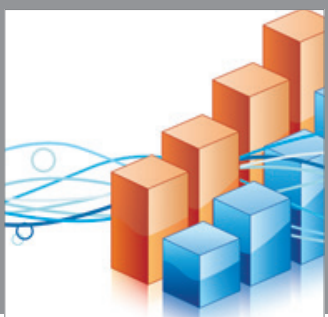

Advances in

Operations Research

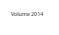

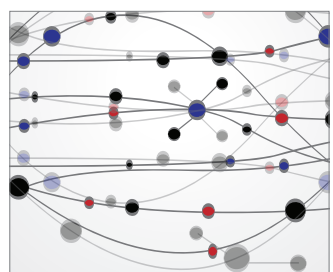

\section{The Scientific} World Journal
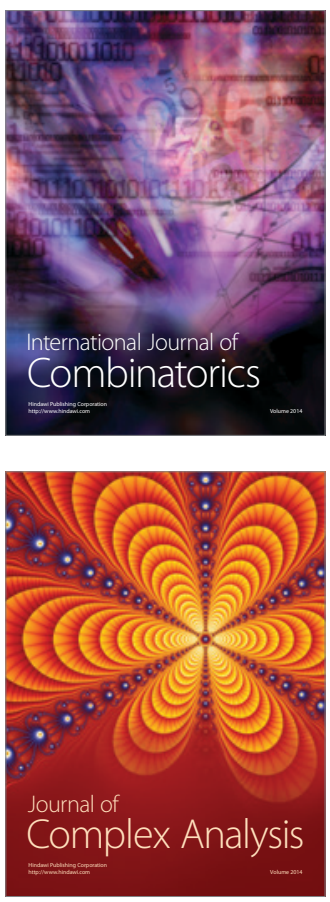

International Journal of

Mathematics and

Mathematical

Sciences
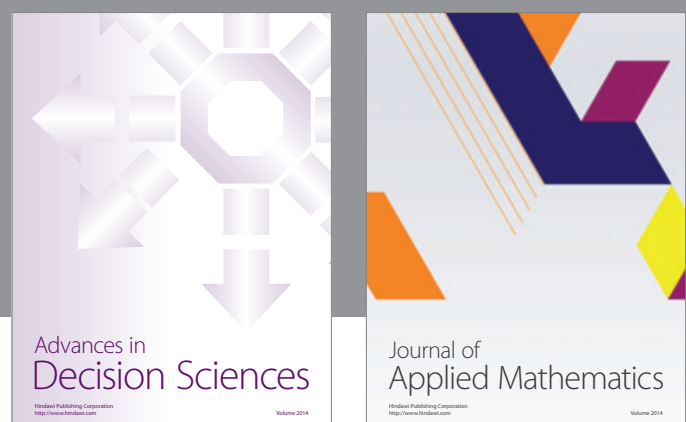

Journal of

Applied Mathematics
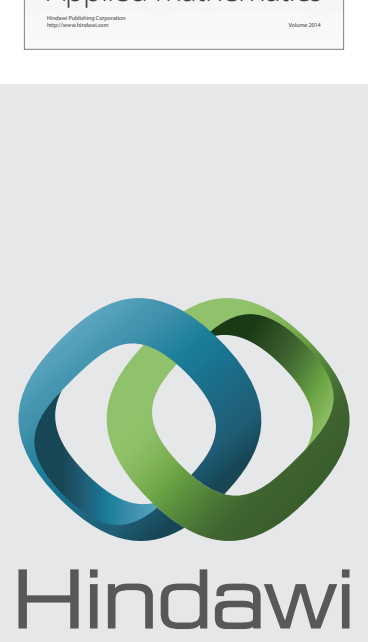

Submit your manuscripts at http://www.hindawi.com
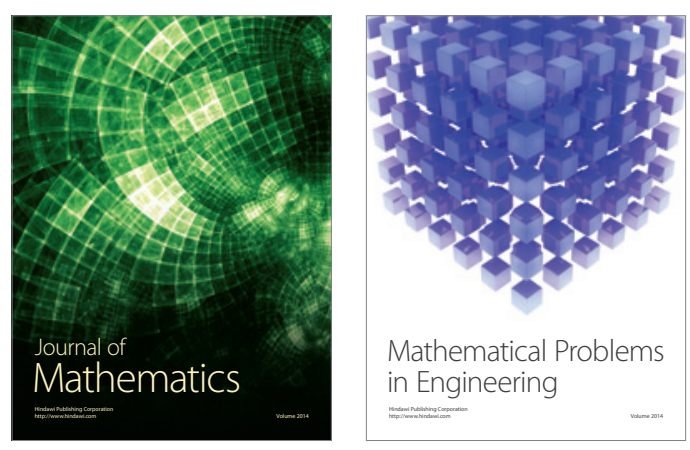

Mathematical Problems in Engineering
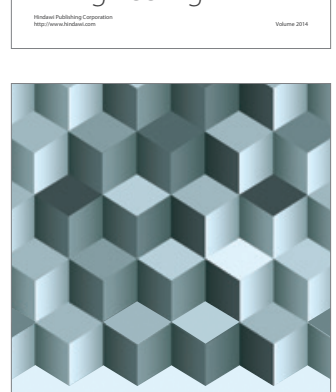

Journal of

Function Spaces
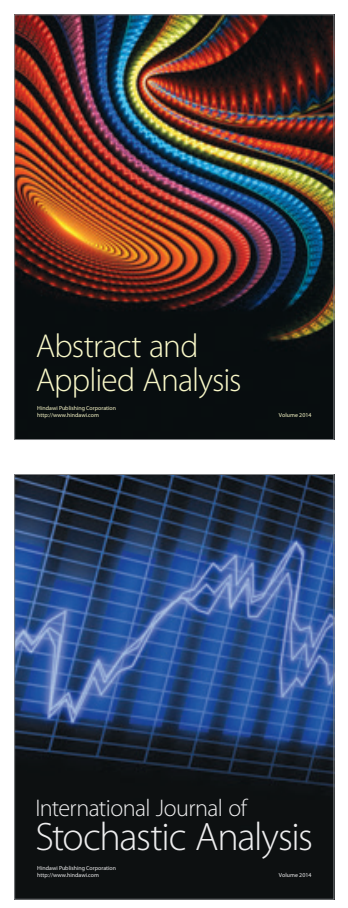

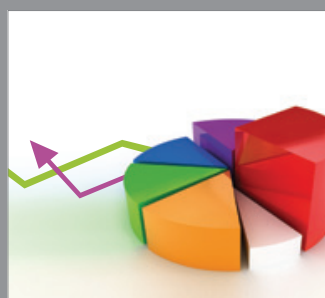

ournal of

Probability and Statistics

Promensencen
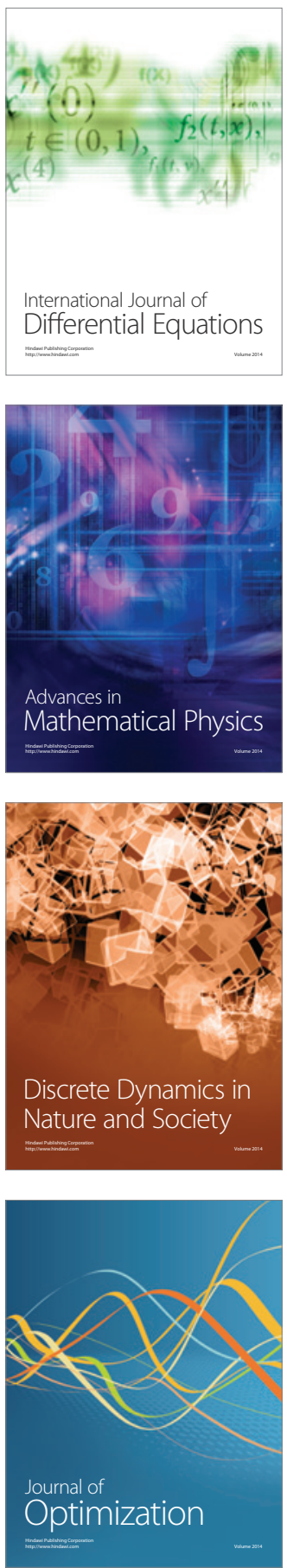